\title{
Las capacidades institucionales \\ en el marco de la descentralización fiscal de los gobiernos sub nacionales en Uruguay: un análisis de tres gobiernos frenteamplistas
}

Institutional capacities for fiscal decentralization in Uruguay's sub-national governments. An analysis of three freanteamplistas departmental governments.

\author{
Martín Freigedo Pelaéz \\ Doctorando en Investigación en \\ Ciencias Sociales con mención en \\ Ciencia Política, FLACSO, sede \\ México. \\ marfreigedo@gmail.com \\ martin.freigedo@flacso.edu.mx
}

\section{Resumen}

Este artículo analiza las capacidades institucionales en materia fiscal de tres gobiernos sub nacionales en Uruguay (Canelones, Rocha y Maldonado) desde el 2005 a la fecha, tratando de relevar sus fortalezas y debilidades a la hora de gestionar sus políticas fiscales. Para este cometido, se discute sobre cuatro factores que condicionan la capacidad institucional en el marco de la descentralización fiscal en Uruguay: estructura interna e innovación de la gestión, recursos humanos, relaciones intergubernamentales e intensidad democrática. Como resultado, se puede observar como el fortalecimiento de ciertos factores ha incidido en un mejor comportamiento en la política fiscal de los casos analizados, aunque no todos los casos obtienen el mismo resultado. También se revela la importancia de la interacción de los diversos factores sobre los avances en las capacidades institucionales.

Palabras claves: Uruguay - descentralización fiscal - capacidades institucionales - gobiernos sub nacionales.

\section{Abstract}

This article analyzes the institutional capacities in the fiscal way of three sub-national governments in Uruguay (Canelones, Maldonado and Rocha) from 2005 to this part, trying to know their strengths and weaknesses. For this

purpose, we discuss fourfactors that determine the institutional
Fecha de aceptación

4.9 .13
Fecha de recepción

7.6 .13 
capacity within the framework of fiscal decentralization in Uruguay: internal structure and innovation management, human resources, intergovernmental relations and intensity democracy. As a result, it can be seen as strengthening certain factors have an impact on a better performance in the fiscal policy of the cases analyzed, although not all cases are equal. Also revealed the importance of the interaction between various factors on the progress of institutional capacities.

Keywords: Uruguay - fiscal decentralization - institutional capacities - sub-national governments

\section{Introducción ${ }^{1}$}

Las elecciones del 2005 significaron un cambio radical en la historia política del Uruguay. Además del primer triunfo electoral de un partido de izquierda a nivel nacional, éste también obtuvo la victoria en ocho de los diecinueve Gobiernos Departamentales (GDs).

Estos cambios también se expresan en nuevas formas de acción política. Así, las capacidades institucionales de los gobiernos sub nacionales frenteamplistas se volvieron una incógnita. Sobre todo en referencia a las capacidades que pudieran asumir en algunos temas fundamentales para la gestión de políticas públicas a nivel local, como la cuestión fiscal. En este sentido, este artículo busca responder a la siguiente pregunta: ¿Qué capacidades institucionales tienen los gobiernos sub nacionales uruguayos en el marco de la descentralización fiscal existente?

El debate político sobre la descentralización ocupa desde hace varias décadas un lugar privilegiado en la agenda sobre la reforma del Estado y las nuevas formas de gestión de políticas públicas. Algunos actores advierten sobre la importancia que asume este mecanismo como instrumento para profundizar la democracia y para mejorar la gestión; pero sobre todo para superar las principales carencias que presentan los sistemas políticos latinoamericanos y su fuerte centralización de poder.

Uno de los temas más importantes y problemáticos a la hora de analizar los procesos descentralizadores tiene que ver con la distribución de recursos financieros entre los diferentes niveles de gobierno. La discusión sobre qué tantas responsabilidades pueden asumir los gobiernos sub nacionales a la hora de cumplir con su función fiscal es muy variada, algunos asumen una postura radical al creer que la gran mayoría de los recursos financieros deben estar en manos de los gobiernos locales, mientras que otros adoptan 
una postura centralista arrogando que la responsabilidad fiscal debe estar en manos de los gobiernos centrales. Sin embargo, estos planteos tienden a ser normativos, olvidando las capacidades de los gobiernos sub nacionales para hacer frente a dichas responsabilidades fiscales.

Tomando en cuenta esta premisa, es que este artículo tiene cuatro cometidos fundamentales. En primer lugar propone una revisión del concepto de descentralización fiscal y de sus alcances y limitaciones para el análisis, como también presentar brevemente el modo en que la descentralización fiscal se estructura en el caso uruguayo.

En segundo lugar, se trabaja sobre el marco metodológico del artículo; a su vez aborda el concepto de capacidades institucionales, analizando los factores que pueden incidir para determinar las mismas en el marco de la descentralización fiscal. Este trabajo está centrado en cuatro factores determinantes: la estructura interna y la innovación de la gestión, los recursos humanos, las relaciones intergubernamentales (RIGs) y la intensidad democrática.

En tercer lugar se intenta estudiar el comportamiento de estos factores en tres casos particulares: Rocha, Maldonado y Canelones, desde el 2005 a 2012.

Por último, en la sección de conclusiones se muestra que si bien han existido ciertos avances en el fortalecimiento de las capacidades institucionales, no todos los casos han tenido el mismo comportamiento en la recaudación de impuestos desde el 2005 al 2012. Mientras que en los casos en donde el punto de partida de las capacidades institucionales era más deficiente (Rocha y Canelones) se observa un crecimiento mayor en la recaudación que en Maldonado, en donde se dio una suerte de inercia en relación a lo realizado por los gobiernos anteriores, debido a los buenos resultados obtenidos.

\section{El proceso de descentralización fiscal: costos y beneficios.}

Se entiende aquí por descentralización el traspaso de atribuciones y responsabilidades de los órganos estatales del nivel central a los subnacionales, dotándolos de mayor autonomía de gestión, de recursos y de capacidad en la toma de decisiones (Arocena, 2008). La descentralización debe ser comprendida como un proceso, y no todos estos procesos son iguales, hay diferentes tipos que marcan el grado de descentralización que se logre alcanzar.

En este sentido Manor (1999), distingue tres tipos de descentralización: (i) desconcentración o descentralización administrativa, (ii) descentralización fiscal, (iii) descentralización o devolución democrática.

La primera refiere a la cesión de atribuciones en materia de gestión de líneas de acción pública desde esferas centrales a los niveles de gobierno jerárquicamente subordinados 
o menor alcance. Este tipo de descentralización adquiere un carácter exclusivamente operativo ya que se corresponde al traspaso de actividades específicas asociadas sobre todo a la implementación de un determinado programa. La segunda remite a las posibilidades que brinda el gobierno central de intervenir en la definición y ejecución del presupuesto o en las decisiones financieras a los ámbitos sub nacionales. En otras palabras, la administración nacional abre espacios para que los agentes locales influyan en la discusión sobre cómo y dónde utilizar los recursos públicos. Por último, el tercer campo de descentralización supone el traslado de recursos financieros y poder de decisión, ya sea de una política pública y/o de una serie de funciones públicas estratégicas para el desarrollo socioeconómico y político a los gobiernos sub nacionales, promoviendo grados específicos de autonomía de las esferas locales. A su vez también, se asocia a la promoción de la participación de la ciudadanía, por medio de la devolución o la ejecución de las acciones públicas.

\section{Cuadro $N^{\circ}$ 1: Tipos de descentralización}

\begin{tabular}{|c|l|}
\hline $\begin{array}{c}\text { Tipo de } \\
\text { descentralización }\end{array}$ & \multicolumn{1}{c|}{ Atribuciones } \\
\hline Administrativa & Cesión de atribuciones en materia de gestión. \\
\hline Fiscal & $\begin{array}{l}\text { Definición y ejecución del presupuesto o en las decisiones } \\
\text { financieras a los ámbitos sub nacionales. }\end{array}$ \\
\hline Democrática & $\begin{array}{l}\text { Participación de la ciudadanía, por medio de la devolución o la } \\
\text { ejecución de las acciones públicas. }\end{array}$ \\
\hline
\end{tabular}

Fuente: elaboración propia en base a Manor (1999) y Falletti (2003)

En las páginas que siguen nos centraremos en el segundo tipo, es decir, la descentralización fiscal, entendiendo por tal una distribución de competencias en materia fiscal (ingreso y gasto) entre diferentes niveles de gobierno, que determina cuánto corresponde recaudar a cada nivel gubernamental y cómo debe de gastarlo, cuestión que suscita mucha controversia.

De manera general puede afirmarse que cuantos mayores sean las transferencias de competencia, mayor será el grado de descentralización alcanzado. En este sentido entra en juego, y desde un lugar fundamental, el sistema fiscal que se establezca para determinar el manejo de recursos financieros de las estructuras locales. Así: "la descentralización fiscal ocurre cuando las competencias fiscales (ingresos y gastos públicos) se desplazan del nivel superior o central hacia el inferior o subnacional y constituye por lo general un proceso dirigido desde el nivel central.” (Aghón y Krause-Junk, 1993:8) 
Cuando se analiza la composición de un sistema fiscal y el grado de descentralización que puede alcanzar, toman partido una gran cantidad de factores políticos, sociales y económicos que establecen qué tan conveniente o no para el funcionamiento del sistema de gobierno es la trasferencia de competencias y recursos. Cada caso es particular y no se puede establecer ciertamente un patrón común, sin embargo la división del sistema fiscal en la clásica categorización de Musgrave (1991) -estabilización, distribución y asignación- ayuda por lo menos a tener un panorama generalizado de qué rol cumplen en la mayoría de los casos el gobierno central y los gobiernos subnacionales.

Usualmente se establece que las funciones de estabilización y distribución están en manos de los gobiernos centrales, mientras que la literatura establece como competencia de los gobiernos subnacionales la de asignación, entendida como la forma de ordenar y establecer de manera adecuada los recursos para garantizar la oferta y la accesibilidad a los bienes y servicios públicos. La tesis general es que dentro de cada país las necesidades son heterogéneas, y cada localidad tiene sus propias preferencias, y en este sentido

los respectivos gobiernos locales provean los niveles de producto Pareto-eficientes a sus respectivas jurisdicciones que la provisión por el gobierno central de cualquier nivel prefijado y uniforme de producto para todas las jurisdicciones (Oates, cit. en Aghón y Krause-Junk, 1993:9)

Otro de los autores clásicos que defiende la tesis de la descentralización fiscal es Samuelson (1954), tomando como punto de partida la satisfacción de los individuos a la hora de adquirir servicios públicos. Este autor pone énfasis en separar por un lado los bienes privados de los públicos. En relación a los primeros, plantea que responden a los intereses individuales por medio del sistema de mercado, ya sea tanto en grandes metrópolis como en pequeñas comunidades. Sin embargo, en el caso de los servicios públicos, responden a preferencias agregadas, a los intereses de una comunidad y no los de un individuo; esto tiene como consecuencia la insatisfacción de los individuos con los servicios públicos cuando se brindan en grandes dimensiones territoriales porque priman intereses individuales y no colectivos.

Si bien la asignación es la principal función de los gobiernos subnacionales, para poder cumplir con esta función existen diferentes formas de transferencias de competencias y responsabilidades en los ingresos y gastos, desde una amplia capacidad de decisión de los gobiernos locales hasta el otro extremo donde la capacidad de decidir está fuertemente restringida, y "la competencia fiscal del nivel inferior o subnacional puede reducirse mediante disposiciones que hagan obligatoria la prestación de determinados servicios” (Aghón y Krause-Junk, 1993:10). 
Por tanto, si se analizan las diferentes formas de capacidad de decisión que tiene los gobiernos subnacionales sobre los ingresos y gastos, que están directamente vinculados con las limitaciones y restricciones que el gobierno central impone, se puede observar una amplia gama de procesos de descentralización fiscal -en las próximas páginas, se definen las principales características del sistema fiscal descentralizado en Uruguay-.

Por último, existen diferentes posiciones en cuanto a los beneficios o costos que puede tener como consecuencia el hecho de dotar de mayores potestades fiscales a los gobiernos de nivel inferior. En este sentido, si se plantean los posibles beneficios, el primero tiene que ver con la eficiencia económica y la posibilidad de cubrir las demandas ciudadanas. Así Cabrero platea

los servicios públicos son más eficientes si se relacionan con las necesidades y preferencias de la población que los demanda, dado que el proveerlos en el espacio local permite ofrecerlos a menor costo, se aprovechan mejor las economías de escala, y se fortalecen las responsabilidades fiscales de los gobiernos subnacionales (Cabrero, 2006:2)

Uno de los argumentos más citados para referirse a las bondades de la descentralización fiscal pone énfasis en la transparencia a la hora de utilizar los recursos públicos. El hecho de que los ciudadanos se encuentren más cercanos a las autoridades públicas y a los funcionarios de la burocracia, hace que se presenten mayores espacios de rendición de cuentas.

Otra de las posibles ventajas, es que la cercanía anteriormente mencionada también favorece a la recaudación de impuestos y tasas. Así,

puede mejorar la eficacia en la asignación de los recursos y la multiplicidad de preferencias individuales y la equidad, mediante la vinculación más estrecha de los beneficios de los servicios y sus costos (Aghón y Krause-Junk, 1993:21).

En cuanto a los costos, uno de los argumentos más fuertes sostiene que estos mecanismos de distribución de recursos pueden generar problemas macroeconómicos, que ponen en riesgo la estabilidad económica del país. Las decisiones respectivas recaen en una variedad de actores, haciéndose difícil establecer un patrón de comportamiento macroeconómico a nivel nacional. Otro argumento crítico apunta a las desventajas que se generan en el interior de cada Estado-nación, creándose disparidades en términos de ingresos. Para prevenir esta situación, se sugiere la puesta en marcha de sistemas de transferencias a las unidades subnacionales más desfavorecidas por la descentralización (Oates, 1972). 


\section{La estructura financiera de los Gobiernos Departamentales}

Uno de los grandes problemas que tienen los gobiernos sub nacionales para llevar adelante procesos de políticas públicas a nivel local es la falta de recursos financieros y el caso uruguayo no escapa a esta realidad.

En este sentido, las posibilidades que tienen los GDs en este país para obtener recursos pasan por dos vías: los ingresos directos por medio de una estructura impositiva existente y las transferencias que puedan obtener del Gobierno Central (GC) y de los organismos internacionales.

En cuanto a los recursos propios, los mismos pueden ser recaudados por medio de impuestos o tasas. Los GDs tienen potestades tributarias definidas fundamentalmente en la Constitución de la República; aunque también se contempla la posibilidad de crear leyes tributarias que se originan en la potestad constitucional del Poder Ejecutivo con el fin de promover la gestión pública de los gobiernos locales.

Entre los tributos más importantes de los GDs se puede destacar la contribución inmobiliaria urbana y suburbana, la contribución inmobiliaria rural y la patente de rodados ${ }^{2}$. A su vez, otra de las fuentes de ingresos directos de los GDs está en las tasas que cobran por brindar ciertos servicios públicos. Se pueden diferenciar entre tasas administrativas, tasas servicios remunerados y autorizaciones, tasas por higiene y salubridad y tasas por seguridad y protección.

Los GDs también obtienen ingresos por las transferencias del gobierno central. Si se analizan las transferencias en el caso uruguayo, se puede encontrar un escenario heterogéneo. En primer lugar porque la dependencia no siempre fue igual; se ha ido incrementando paulatinamente. En segundo lugar, si bien la mayoría de las transferencias son no condicionadas, algunas de ellas lo son.

El principal aporte en materia de transferencias no condicionadas surge del artículo 479 de la Ley 17.930. Esta norma establece que se le otorgará a los GDs un total del 3,3\% del total de los recursos del presupuesto nacional. Las transferencias condicionadas derivan de los tributos de origen nacional. Así, según el artículo 485 de la Ley 17.930, el $11 \%$ de los tributos recaudados fuera del Departamento de Montevideo será utilizado para la implementación de políticas públicas en el nivel local.

En la década de 1980 el promedio de las transferencias rondaba entre el 1 y el 1,5\% de los ingresos departamentales. Esta situación ha ido cambiando paulatinamente, hasta llegar a una dependencia mayor, que tiene que ver con la asunción de hecho y no de derecho, de nuevas funciones por parte de los GDs. El siguiente cuadro resume brevemente la importancia que asumen las transferencias en los ingresos departamentales en los últimos años. 


\section{Cuadro $\mathrm{N}^{\mathrm{o}}$ 2: Evolución de los recursos financieros de los GDs}

\begin{tabular}{|l|c|c|c|c|c|c|}
\hline Fuente / Año & 2003 & 2004 & 2005 & 2006 & 2007 & Promedio \\
\hline Transferencias & 30,6 & 31,2 & 33,1 & 34,2 & 33,6 & 32,6 \\
\hline Impuestos & 44,3 & 42,7 & 41,5 & 43,2 & 41,9 & 42,7 \\
\hline Tasas & 16,1 & 15,8 & 17,5 & 15,9 & 16,4 & 16,3 \\
\hline Otros & 9 & 10,3 & 7,9 & 6,7 & 8,1 & 8,4 \\
\hline Total & 100 & 100 & 100 & 100 & 100 & 100 \\
\hline
\end{tabular}

Fuente: Aguilar, 2009.

\section{Apartado metodológico}

En este apartado se plantean la justificación del tema y la selección de los casos, así como el objetivo de la investigación, el diseño de la misma y las fuentes de recolección de información.

En cuanto a la justificación de la elección del tema, hay que destacar que la descentralización en relación a las capacidades institucionales de los gobiernos departamentales en materia fiscal no ha sido un asunto estudiado en profundidad en Uruguay. Generalmente, desde la ciencia política en particular y de las ciencias sociales en general, el proceso descentralizador ha sido abordado desde el punto de vista de la participación y el desarrollo local, o incluso desde la perspectiva de los sistemas electorales; pero no así desde los aspectos institucionales que conforman el sistema fiscal de los gobiernos sub nacionales y la capacidad que los mismos tienen para lograr una mejor gestión en esta arena de política.

A su vez, esta investigación tiene la particularidad de centrarse en tres casos del interior del país que cuentan con gobiernos frenteamplistas. Como se mencionó anteriormente, esto es un hecho inédito en el Uruguay, debido a que excluyendo el caso de Montevideo, el Frente Amplio nunca en su historia había logrado gobernar ninguna intendencia departamental hasta el año 2005.

Por tanto, la relevancia de analizar el funcionamiento de los gobiernos frenteamplistas en el interior no es menor. Se estudian tres gobiernos sub nacionales que presentan ciertas similitudes y también diferencias. En relación a las similitudes, los tres casos son los únicos gobiernos frenteamplistas del interior del país que ganaron las elecciones del 2005 y que lograron mantenerse en el poder al vencer también en las elecciones del 2010.

Por otro lado, hay que partir de la base de que no todos los casos son iguales, y esas diferencias es lo que hace atractivo su análisis. Cuando se analiza la realidad institucional 
de Canelones, Maldonado y Rocha antes del 2005, es posible encontrar bien marcadas estas diferencias. Mientras que Canelones y Rocha se encontraban insertos en una crisis institucional significativa, la realidad del gobierno de Maldonado era totalmente diferente, era una institución estable que contaba con estructuras financieras y administrativas bien definidas.

Así, en primer lugar, la situación fiscal al momento de asumir los nuevos gobiernos en el 2005 era por demás dispar. Mientras que Rocha y Canelones presentaban un fuerte déficit fiscal; en Maldonado se presentaba cierta estabilidad fiscal.

En segundo lugar, en Canelones y Rocha se presentaron prácticas informales en materia fiscal, así como en el manejo de los recursos humanos y su estructura. Un ejemplo claro de esta situación se manifiesta en el incumplimiento en el pago de sueldos que estos dos GDs tenían con sus funcionarios, los atrasos salariales llevaron a prácticas informales que dejaron abierta la posibilidad de que primara el clientelismo y la discrecionalidad. Por último, la consecuencia directa de la crisis institucional fue una desconfianza a nivel de la población que afectó la capacidad de recaudación de los GDs. Tanto en Rocha como en Canelones la situación de desconfianza se vio reflejada luego en los resultados electorales que, como se mencionó, dio lugar a la victoria frenteamplista en ambos departamentos.

Esta situación no se asemeja a la vivida en Maldonado, y si bien existieron cambios a nivel electoral, la estabilidad institucional le permitió de todas formas mantener una cierta estructura recaudatoria.

El objetivo del presente artículo es analizar las capacidades institucionales en materia fiscal en tres gobiernos departamentales de Uruguay durante el período de 2005 en adelante. A su vez, más concretamente se plantean como objetivos específicos:

- Analizar las características del sistema fiscal descentralizado en Uruguay.

- Identificar y analizar cuáles de los factores determinantes señalados en la teoría son relevantes en el caso uruguayo, sus interrelaciones y peso relativo.

En cuanto al diseño de la investigación, se optó por uno de tipo explicativo, y dadas las características del objeto de estudio, se han escogido técnicas de investigación cualitativas, particularmente se utilizaron el análisis de información secundaria con técnica de análisis de datos -leyes, decretos, documentos, estadísticas, informes- y el análisis de información primaria con técnica de entrevista en profundidad a jerarcas públicos tanto del GC como de los GDs y a actores calificados (13 entrevistas en total). 


\section{Buscando un acercamiento al concepto de las capacidades institucionales}

La importancia que asumen las capacidades institucionales en las políticas públicas está fuera de duda. El concepto ha ido transformándose a lo largo del tiempo, destacándose diferentes atributos de acuerdo al período de estudio, a la corriente teórica que se adopta y a las razones por las cuales se estudian las capacidades institucionales. Por tanto, se puede definir como uno de los conceptos "blandos" de las ciencias sociales, que permite una multiplicidad de abordajes y categorizaciones (Ospina, 2002)

La idea de analizar las capacidades de un gobierno para hacer frente a las demandas sociales, tiene un primer centro de atención en la década de 1980, con la intención de objetivar el análisis al logro de un fortalecimiento institucional que impactara también en la reingeniería organizacional. Para esto se apuntaba principalmente a las condiciones administrativas con que las organizaciones podían cumplir o no sus tareas.

Con la reaparición del Estado en la economía y la sociedad, en lo que se denominó la tercera ola de reformas, el tema de las capacidades y las competencias vuelve a ser centro de atención de las investigaciones pero ya desde una perspectiva más compleja;

los problemas provocados por la implementación de programas de ajuste estructural (de tipo neoutilitaristas) $y$ nuevas dudas acerca de si estos bastaban por sí solos para asegurar un crecimiento futuro llevaron a replantear otra vez el papel del Estado. Hacia fines de la década de los 80' comenzó a cristalizar una tercera ola respecto a estas ideas" (Evans, 1996:530). Así, desde mediados de la década del 90'el concepto de capacidades institucionales tomó importancia a la hora de plantear el rediseño de la acción pública luego de las reformas que impulsaron el reduccionismo estatal (Repetto, 2004)

No existe una definición canónica del concepto de capacidades institucionales, sino una amplia gama de enunciaciones que hacen referencia a las mismas. Para este artículo se las define como la aptitud de adaptarse a los cambios de acción administrativa y política con el fin de que, dadas ciertas restricciones contextuales del marco institucional en donde se está inserto, se logre una habilidad tal que se alcance la eficacia y eficiencia a niveles satisfactorios.

El análisis de las capacidades institucionales puede asumir diversas dimensiones. Aquí se distingue entre capacidades administrativas y políticas (Reppeto, 2004; Rosas Huertas, 2008). Esta división tiene un componente analítico que es de utilidad a la hora de 
poder identificar las capacidades institucionales de los gobiernos; ello no obstante, corresponde señalar que, en la práctica, la división entre lo político y lo administrativo no es tajante, sino que los dos aspectos se encuentran mutuamente interrelacionados, y más adelante se demostrará cómo se comporta esta interacción.

En referencia a las capacidades administrativas, la literatura que refería al análisis de las capacidades institucionales hacía alusión principalmente a los componentes que afectan al interior de las organizaciones públicas. Es decir lo referido a las estructuras técnicoburocráticas, limitando el análisis al aparato organizacional. En tanto las capacidades políticas de los gobiernos sub nacionales implican entender que la problemática a la hora de buscar comprender el proceso de descentralización fiscal, no puede limitarse solamente al análisis al interior de una organización. Existe un entorno que condiciona su acción, en el sentido de que "las organizaciones para sobrevivir necesitan intercambiar recursos con su entorno. Necesitan tomar del mundo exterior la materia prima necesaria para funcionar y ofrecer sus productos” (Vergara, 2010:32). Así, los procesos de análisis de las capacidades institucionales implican pensar también en otros componentes que superan lo que la organización a su interior puede mejorar.

\section{5. ¿Cuáles son los factores determinantes de las capacidades institucionales en materia fiscal?}

Tomando en cuenta la gran cantidad de factores determinantes que puede alcanzar el análisis de las capacidades institucionales de un gobierno, se debe decidir cuáles de todos éstos logran ser representativos para explicar el problema público que esta investigación busca retomar. El siguiente cuadro muestra las determinantes que se han seleccionado y sus indicadores. 


\section{Cuadro $\mathrm{N}^{\circ}$ 3: Determinantes e indicadores de la capacidad institucional de los GDs}

\begin{tabular}{|c|c|c|c|c|}
\hline Niveles & Dimensiones & Determinantes & \multicolumn{2}{|c|}{ Indicadores } \\
\hline \multirow{9}{*}{$\begin{array}{l}\text { Micro y } \\
\text { meso }\end{array}$} & \multirow{9}{*}{$\begin{array}{c}\text { Capacidad } \\
\text { administrativa }\end{array}$} & \multirow{3}{*}{$\begin{array}{c}\text { Estructura interna } \\
\text { e innovación en la } \\
\text { gestión }\end{array}$} & \multicolumn{2}{|c|}{ Cambios en la estructura y objetivos } \\
\hline & & & \multicolumn{2}{|c|}{ Mecanismos de innovación en la gestión fiscal } \\
\hline & & & \multicolumn{2}{|c|}{ Competencias y funciones } \\
\hline & & \multirow{6}{*}{ Recursos Humanos } & \multicolumn{2}{|c|}{ Número de funcionarios } \\
\hline & & & \multicolumn{2}{|c|}{$\begin{array}{l}\text { Mecanismos de acceso y ascensos en la función } \\
\text { pública }\end{array}$} \\
\hline & & & \multicolumn{2}{|c|}{ Idoneidad y capacidad para el cargo. } \\
\hline & & & \multicolumn{2}{|c|}{$\begin{array}{l}\text { Formación y capacitación de los recursos } \\
\text { humanos. }\end{array}$} \\
\hline & & & \multicolumn{2}{|c|}{$\begin{array}{l}\text { Capacidad de reclutamiento de expertos en la } \\
\text { materia. }\end{array}$} \\
\hline & & & \multicolumn{2}{|c|}{ Funcionamiento informal de los RRHH } \\
\hline \multirow{6}{*}{ Macro } & \multirow{6}{*}{$\begin{array}{l}\text { Capacidad } \\
\text { política }\end{array}$} & \multirow{4}{*}{$\begin{array}{l}\text { Las Relaciones } \\
\text { Intergubernamentales }\end{array}$} & \multirow{2}{*}{$\begin{array}{l}\text { Canales de } \\
\text { interacción }\end{array}$} & Formales \\
\hline & & & & Informales \\
\hline & & & \multirow{2}{*}{$\begin{array}{l}\text { Dimensiones de } \\
\text { las RIGs }\end{array}$} & Vertical \\
\hline & & & & Horizontal \\
\hline & & \multirow{2}{*}{$\begin{array}{c}\text { Intensidad } \\
\text { democrática: espacios } \\
\text { de rendición de } \\
\text { cuentas }\end{array}$} & Tradicionales & $\begin{array}{l}\text { Espacios de participación y } \\
\text { nivel de la misma. }\end{array}$ \\
\hline & & & $\begin{array}{l}\text { Gobierno } \\
\text { electrónico }\end{array}$ & $\begin{array}{l}\text { Tipo de información: } \\
\text { Ingresos, gastos y deuda. }\end{array}$ \\
\hline
\end{tabular}

Fuente: elaboración propia

Si bien existen otros factores determinantes que pueden incidir y que podrían ser tomados en cuenta, las mencionadas permiten construir un buen recorrido que demuestre las fortalezas y debilidades de las capacidades institucionales de los GDs en materia fiscal en el caso uruguayo. Esta delimitación es necesaria, ya que de otra forma al tratar de abarcar otras determinantes se puede perder de vista el objeto de estudio.

\subsection{Estructura interna de funcionamiento e innovación de la gestión}

La organización administrativa de los gobiernos constituye siempre un desafío para la gestión de las políticas públicas, tanto más en organizaciones relativamente pequeñas como los gobiernos subnacionales, que demandan la cooperación entre una variedad de direcciones o áreas internas, así como su articulación externa con el gobierno nacional. Para los casos seleccionados en esta investigación, toma relevancia la categoría planteada 
por Cabrero (2004) en relación a organizaciones emergentes como consecuencia de nuevas atribuciones en la gestión pública a nivel local; sin embargo las causas del surgimiento de nuevas organizaciones no parecen ser las mismas que las planteadas por este autor. Los cambios en los gobiernos sub nacionales uruguayos tienen que ver con un componente de tipo político - electoral más que de atribuciones de gestión.

Los tres casos a analizar experimentaron cambio de gobierno, y de ser gobernados por partidos tradicionales (Partido Colorado y Partido Nacional), pasaron a ser liderados por un partido que fue haciendo sus primeras armas en materia de gobiernos locales, el Frente Amplio. Es así que estos cambios implicaron modificaciones en las estructuras organizativas anteriores para dar surgimiento a nuevos espacios en donde el tema fiscal fuera entendido de manera diferente, dando lugar a otros procesos, otras jerarquías que invariablemente dieran lugar a cambios en el funcionamiento de la organización, sobre todo vinculado a procesos de modernización de la gestión.

Por otro lado, dentro de las cuestiones que también hacen a la nueva administración y a sus nuevas formas de gestión en materia fiscal, también es importante analizar los mecanismos de innovación que se han implementado.

El concepto de innovación es por demás abordado y discutido, no solo desde las ciencias sociales; sin embargo en esta investigación se tomará como concepto de innovación el planteado por Dávila: "el hacer cosas nuevas, lograr nuevas combinaciones de recursos o hacer las cosas de una nueva manera" (Dávila, cit. Cabrero, Arellano y Amaya; 2007:149). Obviamente este concepto es por demás amplio y puede abarcar una infinidad de cuestiones. Sin embargo, en este trabajo se retoma únicamente para conocer los mecanismos o instrumentos administrativos y de gestión que han buscado la innovación en materia fiscal.

\subsection{Recursos humanos}

Uno de los principales problemas que tienen los gobiernos subnacionales en general y específicamente en el caso uruguayo, es la falta de recursos humanos adecuados para la gestión de las políticas y servicios públicos que deben desarrollar. Por tanto, esta es una de las dimensiones más importantes de las capacidades institucionales con que deben contar los gobiernos departamentales, en especial en cuestiones tan delicadas como el manejo de recursos públicos.

Generalmente se suele afirmar que en los estados que tienden a favorecer el clientelismo y la discrecionalidad en el acceso a los puestos públicos, existe un exceso del número de funcionarios públicos que componen la burocracia. 
Sin embargo, Evans señala que en realidad lo que hay es escasez, en el sentido que

La percepción común en el sentido contrario procede de la tendencia corriente de las organizaciones patrimonialistas a disfrazarse de burocracias weberianas. Abundan los organismos normativos y administrativos, pero la mayoría no tiene la capacidad de perseguir metas colectivas de un modo predecible o coherente, ni el interés de hacerlo (Evans, 1996:524).

El caso uruguayo parece adaptarse a esta definición sobre la estructura burocrática planteada por este autor. Por eso, se debe tomar en cuenta cómo ha funcionado el servicio profesional de carrera en los casos a estudiar, es decir, analizar si realmente existe meritocracia entre los funcionarios que ocupan sus puestos públicos, indagar sobre su perfil profesional y su idoneidad para el cargo, los mecanismos de promoción, los medios de capacitación, las remuneraciones, etc. Esto permitirá conocer qué tan alejados están los gobiernos sub nacionales de los preceptos weberianos básicos de burocracia.

La literatura académica sobre este tema ha tendido a centrarse en las fallas que presenta el servicio civil en la Administración Pública central (por ejemplo Ramos, 2009), dejando de lado el funcionamiento y las prácticas de la burocracia en los gobiernos sub nacionales. Se trata de un tema que también en los estudios académicos sobre Uruguay ha sido soslayado, y que ciertamente requiere especial atención. ${ }^{3}$

Es asimismo necesario prestar atención a los aspectos informales existentes en el desenvolvimiento de los recursos humanos en el ámbito de la administración. Las relaciones humanas dentro de una organización constituyen una determinante para entender el funcionamiento de la misma. Así, se debe destacar que la subjetividad de las personas es un elemento que no se puede descartar, y en esta subjetividad entran una gran cantidad de factores como los son la personalidad, simpatía, ideología, ambiciones, etc. Es decir, no se trata solo de poner en funcionamiento una estructura formal, sino que a su vez hay que lidiar con la incertidumbre de las personas que echan a andar esa estructura (Vergara, 2010).

\subsection{Relaciones intergubernamentales en la descentralización fiscal}

La relevancia del entender el entramado institucional de las RIGs obedece a la dependencia de las autoridades locales uruguayas respecto del gobierno central, en cuanto refiere a la capacidad de aquellas para negociar y coordinar con éste a la hora de definir los recursos con los que van a contar. Siguiendo a Jordana, se entiende aquí por relaciones intergubernamentales 
el establecimiento de sistemas de comunicación, coordinación y toma de decisiones conjunta entre distintos niveles de gobierno, tengan o no un carácter institucionalizado (Jordana, 2001:11).

La complejidad que asume la descentralización fiscal impone la necesidad de la coordinación al interior del Estado, con una acción conjunta entre diferentes organismos formalmente autónomos pero que funcionen de manera interdependiente, sobre todo una coordinación entre los diferentes niveles de gobierno. Por tanto, entender el funcionamiento de las relaciones intergubernamentales es fundamental para analizar las capacidades institucionales de los gobiernos sub nacionales.

La gestión de las RIGs pone en un primer plano la importancia de desarrollar estrategias de negociación entre los diversos organismos gubernamentales involucrados, procurando identificar los puntos de acuerdo esenciales a los que se puede arribar (Isuani, 2005). Este aspecto es clave, ya que la experiencia demuestra que en muchas oportunidades, diferentes organismos estatales se encuentran enfrentados o realizan las mismas tareas sin tener conocimiento de la situación. En otros casos, la labor de un organismo puede estar impidiendo o dificultando la actuación de otro, lo cual duplica los gastos y esfuerzos. También, la falta de coordinación lleva a que el gobierno superior, que por lo general cuenta con los recursos financieros, restrinja la acción de los gobiernos de menor nivel. En este sentido, es necesario recordar una serie de factores que pueden facilitar o dificultar la coordinación intergubernamental. En primer lugar, el sistema institucional que conforma el Estado: mientras algunos diseños prevén mecanismos que facilitan la coordinación, las responsabilidades conjuntas y la descentralización, otros modelos intentan reducir al mínimo la coordinación estableciendo claramente las responsabilidades de cada organismo. En segundo lugar, el contexto político en el que se desarrollan las políticas, esto es: las características del partido de gobierno, la agenda política, la competencia electoral, la opinión pública y las estrategias e intereses de los actores privados; éstos son todos elementos que pueden incidir sobre el tipo de relaciones intergubernamentales que se lleven a cabo y sobre el proceso descentralizador (Garnier, 2000). Algunos autores incluso sostienen la tesis de que la descentralización en América Latina se desarrolló por causas políticas más que por causas económicas y sociales (Finot, 2001). Por otro lado, se pueden presentar dos dimensiones básicas para entender cómo se desarrollan las RIGs: verticales y horizontales (Jordana, 2001). Estas dimensiones son de utilidad para poder clasificar las diferentes formas de interacción entre los gobiernos.

Es común identificar las RIGs como mecanismos establecidos jerárquicamente; empero las interacciones pueden presentarse de manera horizontal, caracterizándose por ser instancias entre gobiernos del mismo nivel. En este caso 
pueden identificarse relaciones de coordinación y cooperación (mancomunidades municipales $y$ provinciales por ejemplo), de conflicto (limitrofes, de competencias, económicas, etc.) y lo que es peor, la ausencia de relaciones que pueden dar lugar, en caso extremo, al conflicto absoluto que se materializa en la incomunicación y consiguiente imposibilidad de lograr acuerdos y consensos (Arandia, 2002:26).

Las RIGs verticales se presentan entre gobiernos de diferentes niveles, entre el gobierno central, los gobiernos intermedios y los gobiernos locales, incluyendo a los tres niveles o solamente a dos de ellos. Es importante tener en cuenta que cuando se habla de RIGs verticales pueden existir diferentes tipos de autoridades que determinan estas relaciones verticales. Siguiendo a Wright (1998) se pueden identificar tres modelos de autoridad vertical en estas interacciones: autoridad coordinada, autoridad inclusiva y autoridad superpuesta.

En el primero de los modelos existe una relación autónoma entre el nivel central y el segundo nivel; pero no de los gobiernos locales, ya que este último depende de alguno de los otros dos, fundamentalmente de los gobiernos intermedios. Por su lado, el modelo de autoridad inclusiva se caracteriza por presentar una fuerte relación con las relaciones jerárquicas; en este sentido, generalmente los gobiernos sub nacionales dependen del gobierno central de manera muy acentuada. Por último, el tercer modelo o de autoridad superpuesta, se basa en relaciones de negociación permanente e interdependencia entre los diferentes niveles. Generalmente este tipo de relaciones se presentan con relación a algunos temas en donde las condiciones jurídicas y administrativas permiten a los niveles sub nacionales ejercer cierta presión sobre los intereses centrales.

Las relaciones entre los gobiernos pueden asumir diversas formas que permiten categorizar los distintos tipos de procesos políticos mediante las cuales se puede desarrollar las RIGs. Generalmente las mismas se presentan mediante canales formales e informales de interacción (Jordana, 2001). Los primeros responden a procesos institucionalizados que se plasman generalmente en normas legales y en consecuencia suelen establecer jerarquías, competencias y mecanismos de sanción (si es que son necesarios). Así, las RIGs de tipo formal

se manifiestan a través de procedimientos de asignación, coordinación y decisión de carácter institucional, centrados en temas sustantivos, de reparto de poderes entre políticas sectoriales y áreas de gestión entre distintos niveles de gobierno, o bien relativos a la distribución de recursos financieros y responsabilidades fiscales (Arandia, 2002:27). 
Por su parte, los canales informales tienden a presentarse en ciertos casos donde las interacciones formales son superadas o complementadas por ciertas prácticas que atraviesan por actores que no están siempre implícitos en los mecanismos formales como pueden ser los partidos políticos.

\subsection{Intensidad democrática}

El marco institucional que conforma una arena de políticas implica la incidencia que tengan los actores públicos y sus canales de interacción, pero también la ciudadanía en general es parte de este conjunto de reglas que hacen al marco en donde se desenvuelven los actores. En este sentido, uno de los puntos importantes que pueden marcar una mejora en la capacidad institucional de los gobiernos locales a la hora del manejo de recursos financieros, tiene que ver con la incidencia que logren los ciudadanos para controlar los gastos del gobierno. Al respecto Cabrero plantea que "la presión por la rendición de cuentas por parte de la sociedad crea, indudablemente, un ambiente propicio para el fortalecimiento institucional local" (2004:12). Incluso, se podría pensar en espacios no solo de control, sino también de intervención en la ejecución o en la evaluación, experiencias locales como los presupuestos participativos son ejemplo de éste tipo de intervención.

La relación entre participación, control y manejo de recursos financieros es fundamental para apreciar las capacidades institucionales de los gobiernos sub nacionales. Las vías de interacción y el grado de incidencia de la participación de los ciudadanos a la hora de la rendición de cuentas pueden variar; en conjunto, constituyen lo que Cabrero (2004) denomina "intensidad democrática".

Los niveles de participación.

La categorización de Mujica (2005), puede ser de utilidad a la hora de entender el tipo de participación en el control de los recursos. Este autor plantea cuatro grados de participación en la gestión pública: participación informativa, participación consultiva, participación decisoria, participación como cogestión / ejecutor.

El primer grado es únicamente de carácter informativo, hay que entender que este mecanismo de participación es básico para que exista cualquier tipo de participación. El objetivo central es brindar información sobre los temas que se estén manejando, este flujo de información es únicamente "top-down" y no existen instancias de negociación o de apertura al diálogo. La participación consultiva es un mecanismo a través del cual la autoridad local decide convocar a la ciudadanía en general o a un grupo determinado con el objeto de conocer la opinión sobre un tema o asunto puntual. El tercer grado, la participación decisoria, implica que la participación además de expresar demandas y puntos de vista también proponee temas al Estado e influye directamente en la toma 
de decisiones. Implica que las decisiones adoptadas por la administración pública no se realicen en función de los intereses de ésta sino de intereses generales definidos políticamente. Por último, el mayor grado de participación en la gestión pública es la participación como cogestión / ejecutor. Este tipo de participación implica la presencia ciudadana en todo el ciclo de la política pública, está presente en la discusión, la elaboración, la implementación y la evaluación de las políticas públicas. Puede pensarse en este tipo de participación en asuntos de carácter social, donde la ciudadanía pasa a ser parte de la ejecución de una determinada política y es un incentivo para una gestión pública eficiente y eficaz.

Los tipos de rendición de cuentas.

Se pueden presentar dos tipos de interacción entre los gobiernos locales y la ciudadanía los tradicionales o de contacto directo y los mecanismos constituidos por las nuevas tecnologías de información.

El primer tipo, de carácter tradicional tiene que ver con prácticas que suelen ser comúnmente utilizadas en los espacios locales de gobierno, que si bien pueden ser denominadas de formas diferentes, en última instancia refiere principalmente al contacto directo entre el gobierno y los actores sociales locales. Los actores participantes en estos espacios pueden variar y ser más abiertos o cerrados; los más abiertos implican una convocatoria amplia, en la que participan desde los jefes de gobierno central hasta los ciudadanos en general. Los espacios más cerrados pueden involucrar una convocatoria restringida, de la que participan actores sociales específicos interesados en la problemática en cuestión. El otro tipo de interacción incorpora elementos que se asocian con las tecnologías de información y comunicación (TICs). En este sentido, se ha puesto en debate la posibilidad que estas nuevas tecnologías, de amplio acceso en Uruguay fundamentalmente por medio de internet, se conviertan en una herramienta muy útil para mejorar los servicios públicos en varios sentidos. De acuerdo a la enunciación e Royo:

1. Transformación de la Administración, haciéndola más eficiente y transparente; 2. Aumento en la calidad de los servicios prestados; 3. Reducción de la burocracia; 4. Mejora de la receptividad y respuesta de las instituciones públicas a las necesidades de los ciudadanos; 5. Mejora de los procesos de rendición de cuentas; 6 . Fomento de la participación ciudadana en debates de tipo político, y 7. Restitución de la confianza ciudadana en las instituciones (Royo, 2008:175).

Esta nueva forma de gestión, denominada gobierno electrónico (e-goverment), puede asumir diferentes funciones para la mejora los procesos de gobierno. Es este es uno de los 
principales puntos que se han desarrollado por quienes ponen énfasis en estos nuevos recursos (por ejemplo Sour-Vargas, 2007:614). En lo que sigue de este trabajo se presta atención a lo que refiere a la rendición de cuentas de los gobiernos sub nacionales en el manejo de recursos financieros. Sin embargo, por lo menos es necesario poner en duda si la cultura en los espacios locales está preparada para asumir estos cambios de gestión y así cuestionarse también el grado de incidencia que pueden tener estos mecanismos de rendición de cuentas. Parece claro que se precisa un cambio cultural para poder adoptar estas nuevas formas, y este cambio se debe desarrollar en dos niveles. El primer nivel requiere un cambio cultural de los ciudadanos para acceder a la información financiera por medio de las TICs; pero es necesario, como señala McIvor (2002), también lograr un cambio cultural al interior de las organizaciones.

Por tanto, es importante analizar si la cultura interna de la organización está dispuesta a rendir cuentas a la ciudadanía por este medio. Se pueden encontrar tres grandes tipos de información en materia financiera por medio de estos nuevos mecanismos de rendición de cuentas: ingreso, gastos y deuda pública (Sour-Vargas, 2007).

\section{Las capacidades institucionales en los casos de Rocha, Canelones y Maldonado}

En esta sección se presentan brevemente algunos de los avances en el fortalecimiento de las capacidades institucionales en estos tres casos desde el 2005 en adelante, así como también las debilidades que aún persisten y que son un problema para mejorar el comportamiento fiscal. Lo que de aquí en más se analiza son las acciones de gobierno tomadas por los tres GDs a partir del período de gobierno iniciado en el 2005, con el propósito modificar la situación presentada y fortalecer las capacidades institucionales.

\subsection{La diferencia en los objetivos y los avances en materia de innovación de la gestión}

En este punto, se debe destacar que si bien las tres Direcciones analizadas dentro de cada GD tienen un mismo cometido (control de ingresos y egresos financieros), los objetivos perseguidos han sido muy distintos.

En Maldonado, el objetivo primordial pasaba por mantener la estructura existente, tratando de recuperar la base tributaria que se había perdido como consecuencia de la crisis de 2002. En los otros dos casos el punto de partida era completamente diferente, lo que lleva a que los objetivos también fueran disímiles. Así, el primer objetivo era volver a poner en funcionamiento la ineficiente maquinaria de recaudación, y en segundo lugar reducir los altos pasivos con los que se enfrentaron al asumir funciones en el 2005. Para cumplir con estos cometidos, resultaba necesario incorporar mecanismos de innovación en la gestión. Así, entre los tres GDs destacan por lo menos seis herramientas. En primer lugar, en los tres casos se logró descentralizar el cobro de tributos, dando a 
los contribuyentes la posibilidad de pagar sus impuestos en oficinas privadas de cobro. En segundo lugar, también en los tres casos se incorporaron nuevos sistemas contables, lo que permitió acceder a la información con mayor detalle y en forma desagregada. En tercer lugar, las nuevas tecnologías de la información y las nuevas herramientas de innovación en la gestión, lo mismo que los sistemas contables o la descentralización de tributos, hacen necesario que los GDs cuenten con la infraestructura informática necesaria para acompañar a dichos procesos de modernización. Así, en los tres casos se incorporaron nuevos procesos y equipos.

En cuarto lugar se debe destacar una de las herramientas utilizadas por la Intendencia de Canelones. Una de las principales trabas para gestionar nuevas políticas a nivel local con la que se enfrentaba este GD era su alto pasivo financiero. Ante esta situación se decidió utilizar un mecanismo innovador que no había sido nunca instrumentado por ningún GD: la emisión de títulos de deuda cotizables en bolsa de valores. ${ }^{4}$ En quinto lugar, y en lo que refiere a los departamentos de Rocha y Canelones, la actualización del catastro departamental es quizás la herramienta de modernización más importante alcanzada desde el 2005 hasta la fecha. ${ }^{5}$ En convenio con el Banco Interamericano de Desarrollo (BID), la Dirección Nacional de Catastro y la Dirección Nacional de Registro, ambos GDs han avanzado en la creación de sendos nuevos catastros departamentales. Finalmente, con el fin de enfrentar el tradicional problemas de la morosidad estructural delos contribuyentes - particularmente los grandes propietarios de tierras-, los tres GDs optaron por facilitar el pago eliminado multas y recargos - mecanismos convencionales que habían demostrado ser ineficaces- y se negociaron de manera diferenciada y específica con los grandes deudores, logrando acuerdos importantes para regularizar la situación.

\subsection{Los escasos recursos humanos capacitados en materia fiscal}

En cuanto a los recursos humanos se destacan por lo menos tres avances. En primer lugar, con la intención de quebrar la discrecionalidad y el clientelismo, se introdujeron mecanismos de oposición y mérito en el acceso y ascensos a cargos públicos departamentales. En segundo lugar, por medio de convenios con organismos internacionales y el gobierno central, se crearon diversos mecanismos de capacitación para los funcionarios. Estos dos elementos refieren fundamentalmente a las características formales de los recursos humanos, pero también se introdujeron algunos cambios referidos a los aspectos informales, en particular los referidos al liderazgo.

El caso de Maldonado es quizás el menos destacable en este aspecto pues, como ya se mencionó, la estructura anterior a 2005 daba lugar a que los funcionarios tuvieran claras sus funciones, sus competencias y sobre todo, los objetivos de la organización. En cambio en los casos de Canelones y Rocha se pueden mencionar por lo menos tres aspectos en donde las transformaciones en la calidad del liderazgo han incidido favorablemente. En primer lugar, los directores de las áreas financieras de ambos GDs 
se propusieron como medida inicial poner fin a las prácticas informales en el manejo del pago de sueldos y recobrar nuevamente la estructura formal para hacer frente a las deudas con los funcionarios. En segundo lugar, se trató de involucrar a los funcionarios en las decisiones y en los objetivos de la organización, generando de esta manera confianza y revalorizando su labor. Por último, los liderazgos también influyen de acuerdo al compromiso que asuman las propias autoridades con los objetivos; en este sentido, en ambos casos, las autoridades cumplieron con este cometido, lo que repercutió positivamente entre los funcionarios.

Sin perjuicio de lo anterior, queda pendiente todavía un asunto de mucha relevancia: la calificación de los recursos humanos. En los tres casos son escasos los recursos de este tipo y existe un serio problema para lograr reclutar profesionales calificados en materia fiscal. Así, parece claro que la debilidad de los recursos humanos es uno de los principales asuntos pendientes. Subsisten numerosos condicionantes para la conformación del funcionariado público departamental. El primero y fundamental es, sin dudas, la discrecionalidad y el clientelismo que han primado en el funcionamiento de la gestión de recursos humanos, lo que permitió que se impusiera una impronta partidaria sobre otra de tipo meritocrática a la hora del acceso a cargos públicos. Esto tiene como consecuencia que no siempre el funcionario que está en el puesto de trabajo es el más capacitado e idóneo para el cargo, con impacto directo sobre la calidad de la gestión. Como resultado de esta situación, los problemas para contar con personal calificado en los GDs son muy grandes.

A su vez, otro problema asociado a esta práctica clientelar es la desconfianza que los propios funcionarios sienten hacia la institución. El saberse parte de un funcionariado poco capacitado, y que por propia experiencia, sabe que está condicionado a los intereses partidarios, provocan la desconfianza hacia lo que pueda pasar con nuevas autoridades en el gobierno. Por tanto, pese a que se han realizado gestiones en los dos últimos períodos de gobierno para capacitar funcionarios y para brindar confianza y estabilidad, persiste en esta cuestión una considerable debilidad.

\subsection{La falta de relaciones intergubernamentales en materia fiscal}

En páginas anteriores se presentaron cuatro tipos de RIGs que se pueden desarrollar en un proceso de descentralización fiscal. Aquí se intentará analizar de manera sintética cuáles son las RIGs desarrolladas.

A la hora de analizar las RIGs de tipo formal y vertical, se debe diferenciar entre las que se orientan a la rendición de cuentas y las que están orientadas a la innovación y modernización de la gestión. En cuanto a las primeras, los GDs deben rendir cuentas al Tribunal de Cuentas de la Nación (TC), y es éste el que aprueba su presupuesto y sus gastos e ingresos. Por otro lado, también se establecieron canales de interacción 
formales entre algunos organismos del gobierno central (especialmente la Oficina de Planeamiento y Presupuesto -OPP- y la Dirección Nacional de Catastro -DNC-) para mejorar las capacidades de gestión de los gobiernos sub nacionales, sobre todo con Rocha y Canelones. Las RIGs de tipo informal y vertical son sin dudas las menos desarrolladas. Se puede destacar algún tipo de relacionamiento entre autoridades centrales de tipo técnico que se tiene con técnicos de los GDs, pero son experiencias muy escasas. En lo que refiere a las RIGs de tipo horizontal y formal, es necesario destacar el rol que cumple el Congreso de Intendentes. Aunque fue creado como un espacio de lobby político para negociar los recursos financieros que el gobierno central les transfiere a los GDs, en los últimos dos períodos de gobierno (desde el 2005 en adelante) se ha transformado en un actor fundamental debido a su intervención en la creación de la patente única de rodados.

Ahora bien, el rol que cumple este organismo sigue siendo de negociación, es decir que el Congreso de Intendentes no tiene ninguna potestad jurídica de establecer acuerdos obligatorios para los GDs en materia fiscal. Por eso, si bien es un avance su intervención en materia de patentes de rodados, no es común que este órgano intervenga sobre el proceso de descentralización fiscal existente.

Por último, también se pueden destacar ciertos canales de interacción entre los GDs pero de tipo informal. Un primer canal desarrollado fue impulsado por la Fundación Liber Seregni y por la Fundación Friedrich Ebert, creando diversos espacios de interacción entre los GDs frenteamplistas, en donde se han intercambiado experiencias en la cuestión fiscal, con exposiciones de algunas experiencias exitosas.

Sin embargo es posible que los espacios más importantes de interrelación pasen por la relación interpersonal entre intendentes y cuadros del gobierno departamental. Un ejemplo es la actualización del catastro departamental. Canelones fue el primer GD en tomar medidas con el fin de mejorar su gestión fiscal por medio de la creación de un nuevo padrón catastral en 2006. Así, a través de canales informales la experiencia fue trasmitida al equipo de gobierno de Rocha y se realizaron las gestiones para realizar el mismo trabajo que en Canelones. Incluso, se logró obtener un similar financiamiento por parte del BID.

Resumiendo, la realidad muestra que si bien se ha avanzado en algunos aspectos de las RIGs instrumentadas en materia formal, sobre todo vinculados a la modernización de la gestión con los convenios firmados entre el gobierno central y los GDs, todavía persisten ciertas debilidades. Resulta necesario establecer espacios formales que mejoren la relación y el vínculo en materia fiscal entre los propios GDs, debido a que el Congreso de Intendentes no está orientado a esta función. Sin embargo, se han suplido algunas de las falencias en materia formal, por medio de un contacto directo entre las propias autoridades de los GDs. 


\subsection{Los mecanismos de intensidad democrática: un factor olvidado}

El último factor determinante de las capacidades institucionales que aborda este trabajo, analiza los mecanismos de participación instrumentados por los GDs en relación a la cuestión fiscal, con el fin de conocer si realmente los mismos han sido útiles para fortalecer las capacidades institucionales departamentales.

En Canelones, Maldonado y Rocha se han puesto en práctica desde 2005 mecanismos de participación y rendición de cuentas que implican el relacionamiento directo entre el gobierno y la ciudadanía. En los dos primeros se crearon órganos de gobierno orientados a la profundización de la descentralización, mediante unidades que tienen como meta fomentar la participación de la ciudadanía, planeando y estructurando los mecanismos orientados a incentivar dicha participación (Cuadro 4).

\section{Cuadro $\mathrm{N}^{\circ}$ 4: Estructuras creadas para fomentar la participación ciudadana}

\begin{tabular}{|c|c|c|c|}
\hline & Nuevas estructuras & Principales funciones & Actores que la integran \\
\hline \multirow{3}{*}{ Maldonado } & $\begin{array}{c}\text { Unidad de } \\
\text { Descentralización }\end{array}$ & $\begin{array}{l}\text { a) Coordinar el proceso de } \\
\text { descentralización en el Dpto. } \\
\text { b) Nexo Junta Local / } \\
\text { Municipios - Gob. Dptal. } \\
\text { C) Incentivar la participación } \\
\text { ciudadana }\end{array}$ & $\begin{array}{l}\text { Gobierno } \\
\text { Departamental }\end{array}$ \\
\hline & Comisión Mixta & $\begin{array}{l}\text { a) Elaborar proyecto de } \\
\text { descentralización junto a la } \\
\text { Unidad de descentralización }\end{array}$ & $\begin{array}{l}\text { Gobierno } \\
\text { Departamental, Junta } \\
\text { Departamental y Juntas } \\
\text { Locales / Municipios }\end{array}$ \\
\hline & $\begin{array}{c}\text { Oficinas } \\
\text { Desconcertadas }\end{array}$ & $\begin{array}{l}\text { a) Nexo vecinos y ONG's - } \\
\text { Gob. Dptal. }\end{array}$ & $\begin{array}{l}\text { Gobierno } \\
\text { Departamental }\end{array}$ \\
\hline \multirow[t]{2}{*}{ Canelones } & $\begin{array}{c}\text { Área de } \\
\text { Descentralización y } \\
\text { participación }\end{array}$ & $\begin{array}{l}\text { a) Coordinar el proceso de } \\
\text { descentralización en el Dpto. } \\
\text { b) Nexo Junta Local / } \\
\text { Municipios - Gob. Dptal. } \\
\text { c) Coordinación de } \\
\text { los Centros Cívicos } \\
\text { d) Incentivar la participación } \\
\text { ciudadana }\end{array}$ & $\begin{array}{l}\text { Gobierno } \\
\text { Departamental }\end{array}$ \\
\hline & Centros Cívicos & $\begin{array}{l}\text { a) Profundizar la } \\
\text { desconcentración } \\
\text { administrativa y la } \\
\text { participación ciudadana }\end{array}$ & $\begin{array}{l}\text { Gobierno } \\
\text { Departamental, } \\
\text { Gobierno Central y } \\
\text { UDELAR }\end{array}$ \\
\hline
\end{tabular}

Fuente: elaboración propia en base a Magri, Freigedo y Tejera (2009) 
Con diferencias en cuanto a los mecanismos instrumentados pero con un mismo objetivo, a raíz de estas estructuras se han incorporado herramientas para la rendición de cuentas y la participación que se basan en canales de interacción entre la ciudadanía y el GD, en donde las autoridades informan sobre los resultados en materia fiscal. En el caso de Rocha, pese a no existir una estructura formal o un programa diseñado con este objetivo, también se han implementado (bien que de manera esporádica y sobre todo ante demandas concretas) canales de rendición de cuentas informativos ante la ciudadanía.

Por otro lado, en materia de gobierno electrónico los avances han sido notorios, sobre todo desde 2005 en adelante con la creación de la Agencia de Gobierno Electrónico y Sociedad de la Información (AGESIC) Esta impronta de desarrollo en el área también alcanza a los GDs; por ejemplo, las 19 intendencias departamentales han creado sitios webs para sus gobiernos. Sin embargo, el avance en gobierno electrónico de los GDs no es homogéneo, el siguiente gráfico resume el alcance del mismo según ciertas categorías. Se puede apreciar que en materia de manejo de información referido al presupuesto y su ejecución, 13 de las 19 Intendencias ofrecen estos datos.

\section{Gráfico $\mathrm{N}^{\mathrm{o}}$ 1: Información y trámites disponibles en los sitios webs de las Intendencias}

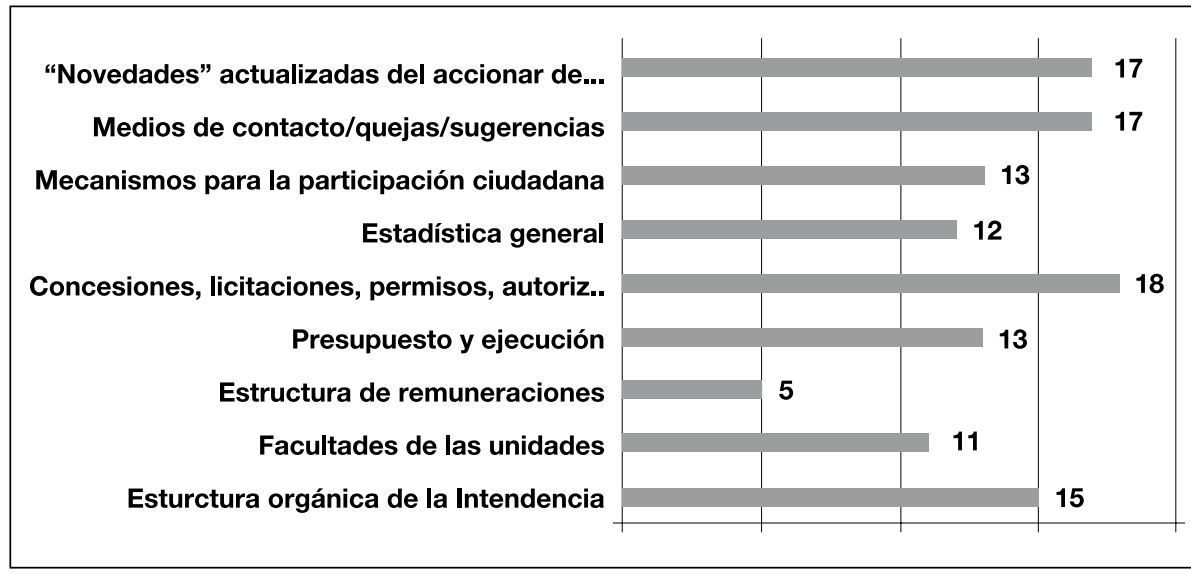

Elaboración propia en base a Cardarello et al (2010)

En los tres casos que aquí se ha trabajado se observa ese avance; en particular, todos incorporaron desde 2005 sitios webs de las intendencias -pero también de las Juntas Departamentales. En los tres sitios se pude encontrar información en relación al manejo de los recursos públicos y todos los GDs analizados presentan los datos básicos: ingresos, gastos y deuda pública. De todas formas, existen diferencias en relación al detalle con que dicha información es brindada. ${ }^{6}$ 
Con los diferentes mecanismos analizados hasta aquí se pueden identificar avances en materia de participación ciudadana y rendición de cuentas en materia fiscal. Se han creado canales de interacción tradicionales, pero sobre todo se han impulsado mecanismos de gobierno electrónico que fomentan la trasparencia y la modernización en la gestión.

\section{La relación entre los factores determinantes y su peso relativo en el marco de la descentralización fiscal}

En el apartado referido a la definición de las capacidades institucionales, se diferenció entre capacidades administrativas y políticas; sin embargo esta diferenciación adquiere un carácter puramente analítico y en la realidad ambas dimensiones se encuentran en continua interacción. De esta forma, es importante también observar la relación existente entre los cuatro factores determinantes analizados, mostrando la interdependencia que existe entre los mismos y cómo no todos adquieren la misma importancia a la hora de analizar las capacidades de los GDs.

\subsection{Los recursos humanos y su relación con los demás factores}

En primero lugar, entre las RIGs analizadas anteriormente se destacaba la importancia y el rol que ha tenido la Oficina de Planeamiento y Presupuesto (OPP) como promotor de proyectos y planes orientados a mejorar la capacidad institucional de los GDs en materia fiscal. Sin embargo, las propias autoridades del organismo central han mencionado la influencia de los recursos humanos a la hora de la ejecución de proyectos en conjunto. Mientras que Rocha y Canelones han sido dependientes de la OPP, Maldonado ha logrado cierta autonomía, y mucho tiene que ver en esto la presencia de recursos humanos capacitados, pero sobre todo funcionarios con tareas claras y precisas.

Por tanto, cuanto mejor capacitados y orientados en las funciones que deben cumplir los funcionarios (y en consecuencia la organización), menor es el apoyo del gobierno central para mejorar sus capacidades; así en Maldonado las RIGs con el GC fueron menos intensas que en los otros dos casos.

A su vez, los recursos humanos impactan en los mecanismos instrumentados para incentivar la participación ciudadana y la rendición de cuentas en materia fiscal. Desde 2005 Canelones y Maldonado destinaron recursos humanos especializados y capacitados para orientar los procesos de participación ciudadana, mientras que Rocha no lo hizo sino hasta el 2011. Por tanto, los GDs que más han avanzado en materia de rendición de cuentas han sido los que destinaron recursos humanos para implementar los mecanismos.

Por otro lado, también los recursos humanos han incidido en la implementación de los mecanismos de innovación. Un ejemplo claro fue lo sucedido con la creación del nuevo catastro en Canelones y Rocha. A raíz de las entrevistas realizadas, las autoridades de 
la Dirección Nacional de Catastro (DNC) reconocen que el trabajo con Canelones se dio de manera fluida y se cumplieron los plazos, debido a que el equipo de trabajo funcionaba acorde a las exigencias que se necesitaban para implementar un nuevo catastro departamental en el tiempo establecido . Sin embargo, en Rocha el trabajo se dio de forma paulatina y aún se sigue trabajando, y mucho tienen que ver en esto los recursos humanos con los que cuenta su Intendencia.

\subsection{El impacto de las RIGs en Canelones y Rocha}

Así como Maldonado ha logrado trabajar con cierta autonomía en relación al gobierno central, en los casos de Canelones y Rocha el apoyo y los canales de interacción de tipo vertical han sido determinantes para mejorar las capacidades administrativas de los GDs. Se acaba de mencionar la influencia de la OPP para la capacitación de funcionarios, así como en el apoyo en la incorporación de nuevas herramientas de modernización. También en este aspecto otro actor fundamental ha sido la DNC.

Por su parte, también los canales de interacción horizontales han sido útiles para la promoción de mecanismos de innovación. La iniciativa en relación a la actualización de catastro en Rocha, se llevó adelante como consecuencia de un intercambio de información con la Intendencia de Canelones.

\subsection{Intensidad democrática y su incidencia en la innovación de la gestión}

Por último, las movilizaciones y manifestaciones que desarrollaron los vecinos de Canelones ante el aumento de la contribución inmobiliaria como consecuencia de la elaboración de un nuevo catastro departamental, han generado que la participación ciudadana impacte en los mecanismos de innovación desarrollados. Así, las reacciones que esta movilización forjó en la Junta Departamental como en el Intendente y su grupo de gobierno, tuvieron como resultado la elaboración de una comisión multipartidaria para discutir sobre las posibles fallas técnicas que el nuevo catastro departamental podría contener. Esta comisión está funcionando en momentos de elaborarse este artículo; y si bien no es posible predecir el resultado de este trabajo, sí se puede afirmar que la presión de la ciudadanía ante un hecho concreto que afecta la cuestión fiscal a nivel sub nacional, ha incidido sobre otro de los factores que afectan a las capacidades institucionales: la innovación en la gestión.

La siguiente figura busca resumir las relaciones entre factores determinantes. Las flechas están dirigidas hacia la determinante que se ve influenciada por otra. Así también, se puede observar la fortaleza de las relaciones en los diferentes espesores de las flechas, es decir, cuanto mayor tamaño presenta la flecha, la relación entre factores es más fuerte. 


\section{Figura $\mathrm{N}^{\circ}$ 1: Relación entre los factores determinantes en el caso uruguayo actual}

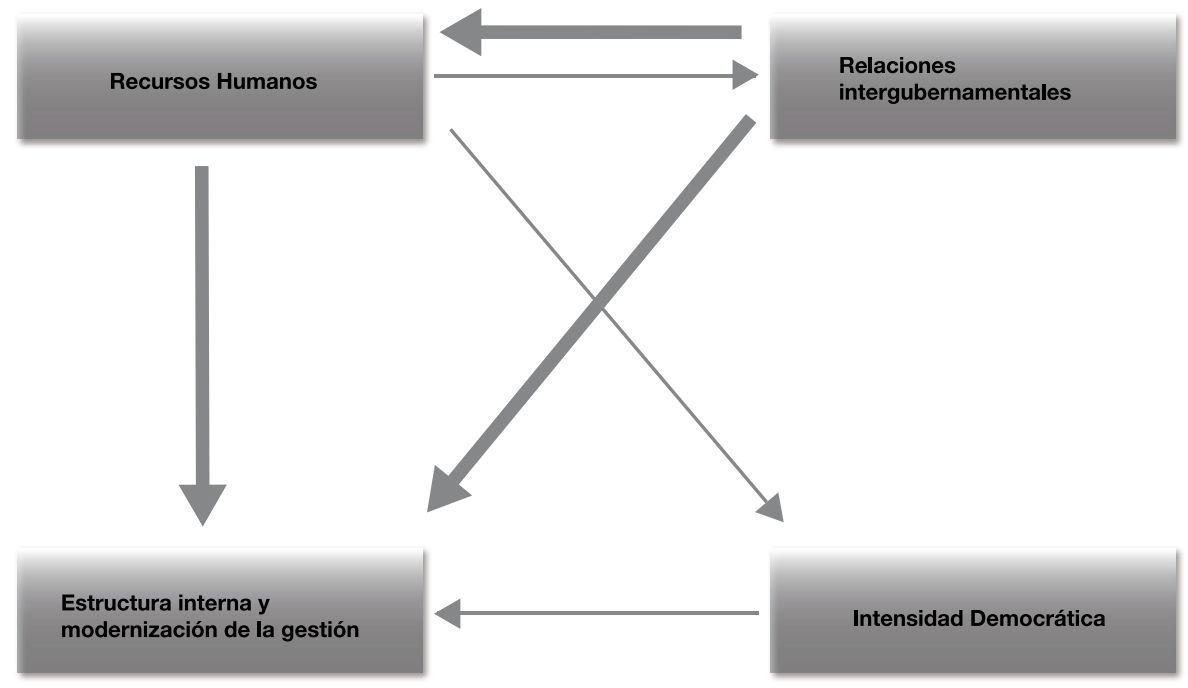

Fuente: elaboración propia

Ahora bien, estas relaciones advierten que no todos los factores adquieren la misma importancia a la hora de definir las capacidades institucionales de los GDs en materia fiscal; se debe diferenciar el peso relativo que adquieren para definir las capacidades institucionales. Así, tanto los recursos humanos como las relaciones intergubernamentales son los factores que generan los mayores círculos virtuosos que impactan de manera positiva a las capacidades institucionales de los GDs en materia fiscal. Estos dos factores influyen de manera significativa en la conformación del marco de la descentralización fiscal.

\section{Conclusiones}

No es novedad que el sistema fiscal descentralizado uruguayo deja poco espacio para la intervención de los GDs y la dependencia hacia el GC es muy fuerte. A su vez, este sistema es todavía poco eficiente y persisten importantes problemas de gestión. En éstos, mucho tienen que ver las debilidades de las capacidades institucionales de los GDs. Así, se han analizado diversas dificultades, tanto de tipo administrativas como políticas, que impactan en la capacidad de recaudación. El siguiente cuadro busca resumir algunas de ellas, junto a recomendaciones que apuntan a mejorar la gestión fiscal. 


\section{Cuadro $\mathrm{N}^{\circ}$ 5: Debilidades y recomendaciones en la gestión fiscal}

\begin{tabular}{|c|c|c|}
\hline Determinante & Debilidades & Recomendaciones \\
\hline \multirow{3}{*}{$\begin{array}{l}\text { Estructura interna } \\
\text { e innovación } \\
\text { en la gestión }\end{array}$} & $\begin{array}{l}\text { No se modificaron las } \\
\text { estructuras internas en } \\
\text { Rocha y Maldonado }\end{array}$ & $\begin{array}{l}\text { Reestructurar en el corto plazo las } \\
\text { Direcciones de Hacienda, dividiendo } \\
\text { las funciones de gastos e ingresos. }\end{array}$ \\
\hline & $\begin{array}{l}\text { Problemas para poner en } \\
\text { práctica los nuevos sistemas } \\
\text { contables }\end{array}$ & $\begin{array}{l}\text { Cursos de especialización para } \\
\text { funcionarios y autoridades en el } \\
\text { manejo del sistema }\end{array}$ \\
\hline & $\begin{array}{l}\text { Atrasos en la actualización } \\
\text { del catastro y del registro de } \\
\text { propietarios en Rocha }\end{array}$ & $\begin{array}{l}\text { Continuar el trabajo junto a Catastro, } \\
\text { estableciendo plazos claros de } \\
\text { finalización }\end{array}$ \\
\hline \multirow{3}{*}{ Recursos humanos } & \multirow{2}{*}{$\begin{array}{l}\text { Clientelismo y } \\
\text { discrecionalidad en el manejo } \\
\text { de recursos humanos }\end{array}$} & $\begin{array}{l}\text { Seguir estableciendo mecanismos de } \\
\text { transparencia en el acceso y ascenso de } \\
\text { los funcionarios }\end{array}$ \\
\hline & & $\begin{array}{l}\text { Crear un sistema de acceso y ascenso } \\
\text { homogéneo para todos los GDs }\end{array}$ \\
\hline & $\begin{array}{l}\text { Falta de recursos humanos } \\
\text { calificados }\end{array}$ & $\begin{array}{l}\text { Establecer convenios con el GC } \\
\text { y organismos internacionales para } \\
\text { promover la incorporación de recursos } \\
\text { calificados y continuar capacitando a } \\
\text { los actuales recursos. }\end{array}$ \\
\hline $\begin{array}{l}\text { Relaciones } \\
\text { gubernamentales }\end{array}$ & $\begin{array}{l}\text { Falta de canales horizontales } \\
\text { formales }\end{array}$ & $\begin{array}{l}\text { Crear marco normativo para promover } \\
\text { las RIGs horizontales entre los GDs } \\
\text { más allá del Congreso de Intendentes }\end{array}$ \\
\hline \multirow{2}{*}{$\begin{array}{l}\text { Intensidad } \\
\text { democrática }\end{array}$} & \multirow{2}{*}{$\begin{array}{l}\text { Debilidad en las políticas } \\
\text { de comunicación que está } \\
\text { condicionada la voluntad } \\
\text { política }\end{array}$} & $\begin{array}{l}\text { Establecer un marco normativo } \\
\text { que promueva la participación y la } \\
\text { rendición de cuentas }\end{array}$ \\
\hline & & $\begin{array}{l}\text { Crear canales donde la participación } \\
\text { en materia fiscal alcance niveles, por lo } \\
\text { menos, de tipo consultiva. }\end{array}$ \\
\hline
\end{tabular}

Fuente: elaboración propia en base a datos del Instituto Nacional de Estadística 
De todas formas es necesario remarcar que desde el 2005 a la fecha, con el cambio de gobierno en los tres casos analizados, se reforzaron algunos aspectos que han lograron mejorar la gestión fiscal sub nacional. Sin embargo estos cambios se ven reflejados de manera diferente de acuerdo a cada GD; los gráficos que a continuación se presentan buscan mostrar el comportamiento en la recaudación en los tres casos analizados de los dos tributos fundamentales: contribución inmobiliaria y patente de rodados.

Gráficos $\mathrm{N}^{\circ} 2$ y 3: Promedio del crecimiento de la recaudación por contribución inmobiliaria y de la patente única en Maldonado para el período $2005 / 2010$ a precios constantes. Base $2005=100 \%$
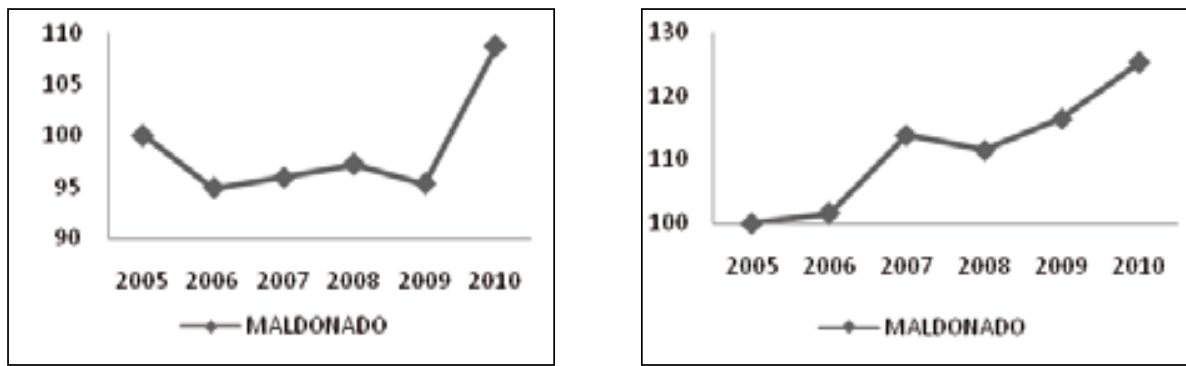

Fuente: elaboración propia en base a datos del Instituto Nacional de Estadística

Gráficos $\mathrm{N}^{\mathrm{o}} 4$ y 5: Promedio del crecimiento de la recaudación por contribución inmobiliaria y de la patente única en Canelones para el período $2005 / 2010$ a precios constantes Base $2005=100 \%$
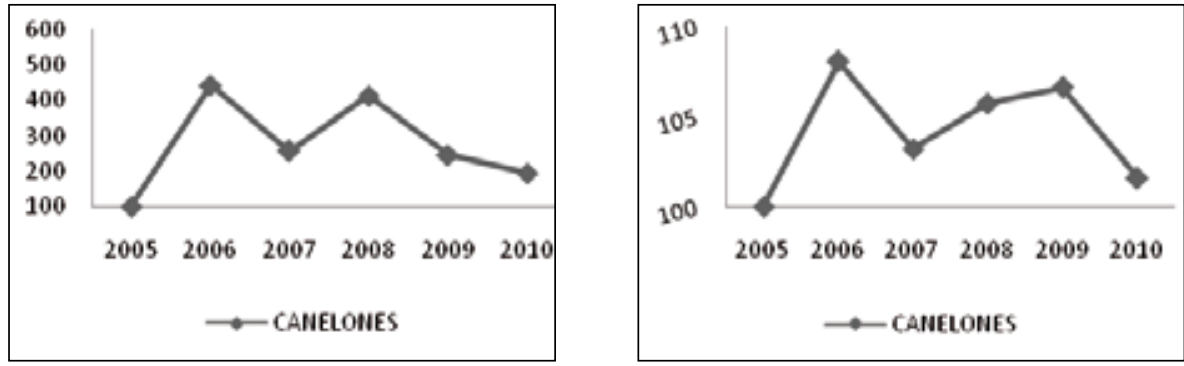

Fuente: elaboración propia en base a datos del Instituto Nacional de Estadística 


\section{Gráficos $N^{\circ} 6$ y 7: Promedio del crecimiento de la recaudación por contribu- ción inmobiliaria y de la patente única en Rocha para el período 2005/2010 a precios constantes. Base $2005=100 \%$}
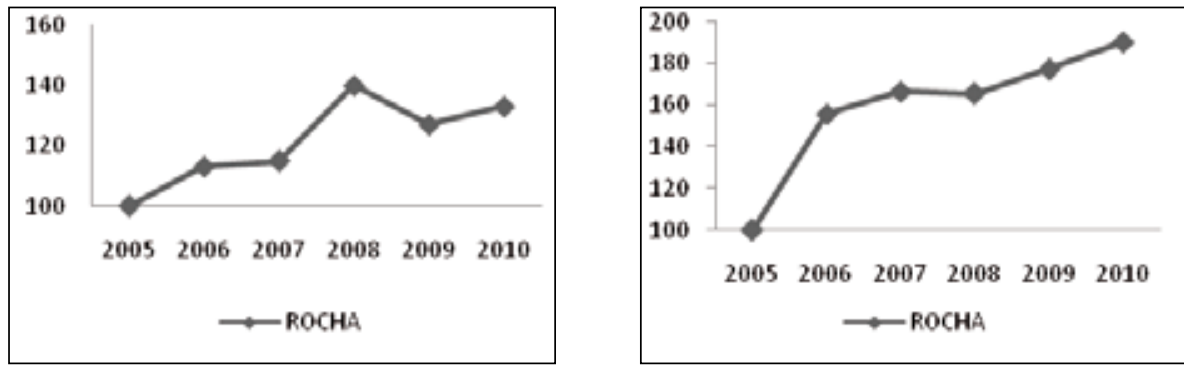

Fuente: elaboración propia en base a datos del Instituto Nacional de Estadística

Por un lado, hay que destacar que Rocha ha tenido un crecimiento constante durante el período 2005-2010. Por su parte, Maldonado no muestra un crecimiento significativo, circunstancia que puede estar asociada a que dicho GD ha mantenido cierta estabilidad institucional y en consecuencia no han existido cambios importantes en la recaudación tributaria.

Quizás Canelones es el caso que más llamativo. De acuerdo a lo ya señalado, este GD presentó avances muy importantes en su fortalecimiento institucional, sin perjuicio de la persistencia de ciertas debilidades que dificultan el mejoramiento del comportamiento fiscal. Las posibles explicaciones a la irregularidad en esta materia en el período analizado pueden estar asociadas a dos razones: en primer lugar, la apertura de canales de recuperación de activos permitió que la recaudación en los años que se incorporaron estos mecanismo haya sido significativamente mayor; por otro lado, las diversas disputas en la fijación de los precios de las patentes de rodados entre los Departamentos afectó de manera significativa a Canelones y Montevideo principalmente, y eso explica el comportamiento en la recaudación de patentes de rodados.

Por otro lado, no todos los GDs tienen las mismas capacidades institucionales, y las diferencias están marcadas también por la trayectoria institucional de cada uno de los casos; en páginas anteriores se mostró que los puntos de partida de cada uno de los GDs eran por demás disímiles, y esto se ve reflejado en las debilidades y fortalezas que presentan en la actualidad cada una de las Intendencias.

También es necesario destacar que los factores analizados están interrelacionados y no se pueden estudiar sin entender que entre los mismos existen dependencias muy fuertes ya que se encuentran insertos dentro de un marco institucional que los condiciona, y en 
consecuencia para el caso uruguayo no todos los factores analizados asumen la misma importancia en la definición de las capacidades institucionales.

Por último, como se planteó al principio de este artículo, las posiciones en contra o a favor de la descentralización fiscal tienden a ser normativas, olvidando muchas veces la capacidad de gestión que tienen los gobiernos sub nacionales en materia fiscal; un asunto por demás relevante a la hora de analizar las políticas públicas a nivel local.

\footnotetext{
${ }^{1}$ Este artículo forma parte de la investigación realizada para obtener el grado de Maestro en Políticas Públicas por la Universidad Autónoma Metropolitana, México. Agradezco los comentarios y sugerencias de la Dra. Myriam Cardozo Brum y de los evaluadores anónimos.

${ }^{2}$ En otros países estas tasas adquieren otros nombres; en México, por ejemplo, serían el equivalente de los impuestos predial y de tenencia.

${ }^{3}$ De todas formas, el análisis sobre la estructura del sistema de carrera y su influencia en la determinación de los recursos humanos no es posible tratarlo de manera profunda en este artículo. Simplemente se busca realizar un breve recorrido que permita conocer de forma general como se dio este proceso.

${ }^{4}$ Con esto, la Intendencia va a pagar con cobranzas futuras, se hizo una sesión de cobranzas a 15 años y hubo inversionistas que compraron y pusieron el dinero para pagar esas deudas. Se creó un fideicomiso en convenció con la Administradora de Fondos de Inversión S.A. (AFISA) perteneciente al Banco de la República Oriental de Uruguay (BROU) para que administre este dinero y sea el encargado de la devolución del dinero a los inversores.

${ }^{5}$ El Catastro Departamental en estos casos tenía una desactualización de casi 40 años. En el caso de Maldonado se ha creado un Catastro propio antes del 2005.

${ }^{6}$ Maldonado y Canelones brindan una información más detallada que Rocha.
} 


\section{Bibliografía}

Aghón y Krause-Junk (1993) Descentralización fiscal: marco conceptual. Naciones Unidas. CEPAL, Santiago de Chile

Arandia, I. (2002) "Descentralización y relaciones intergubernamentales en Bolivia" Revista Convergencia, Vol. 9, número 30: 19-45, Universidad Autónoma del Estado de México. Toluca, México.

Arocena, J. (2008) "Los desafios de la descentralización y la participación ciudadana en el Uruguay". Cuadernos para el Desarrollo Local. Programa ART Uruguay. Montevideo,Uruguay.

Aguilar, J. (2009) Finanzas y gestión de los gobiernos sub nacionales en Uruguay. Banco Interamericano de Desarrollo. Sector de Capacidad Institucional y Finanzas División de Gestión Fiscal y Municipal.

Cabrero, E. (2004) “Capacidades institucionales en gobiernos sub nacionales de México. ¿Un obstáculo para la descentralización fiscal?”. Gestión y Política Pública. XIII (3): 753-78.

(2006) “Los retos institucionales de la descentralización fiscal en América Latina" Reforma y Democracia 34: 2-19, Caracas.

Cabrero, E.; Arellano, D.; Amaya, M. (2007) "Cambio en organizaciones gubernamentales: innovación y complejidad" En Arellano, C; Cabrero, E y del Castillo, A. (comp). Reformando al gobierno. Una visión organizacional del cambio gubernamental. CIDE, editorial Miguel Ángel Porrúa. México, 2007: 139-168.

Cardarello, A.; Freigedo, M.; Vairo, D.; Abraham, M. (2010). "Mucho ruido... y algunas nueces. Los vaivenes de la descentralización en la administración Vázquez (2005-2010)". En: Narbondo y Mancebo (comp.) Reforma del Estado y Políticas Públicas en la Administración Vázquez: acumulaciones, conflictos y desafíos. Editorial Fin de Siglo, Montevideo. Uruguay, 2010: 54-74.

Cardarello, A.; Magri, A.; Mancebo, E.;Vario, D. Línea de base del proyecto “Apoyo a la cohesión social y territorial. Uruguay Integra" (Programa Uruguay Integra). Convenio OPP/ICP-UDELAR

Díaz, M. (2002): "Federalismo fiscal y asignación de competencias: una perspectiva teórica”. Economía, Sociedad y Territorio, Vol. III, Núm. 011: 387-407, enero-junio, 2002. El Colegio Mexiquense, A.C. México

Evans, P. (1996) “El Estado como problema y como solución”, Desarrollo Económico. Revista de Ciencias Sociales, enero-marzo de 1996, vol. 35, núm. 140: 529-562, Instituto de Desarrollo Económico y Social.

Falletti, G. (2003): "A sequential theory of decentralization and its effects on the intergovernmental balance of power: latinamerican cases in comparative perspective”, en: http://kellogg. nd.edu/publications/workingpapers/WPS/314 Con acceso en agosto y setiembre de 2009. 
Finot, I. (2001): Descentralización en América Latina: teoría y práctica. ILPES/CEPAL. Santiago.

Garnier, L. (2000). Función de coordinación de planes y políticas. CEPAL. Serie: Gestión Pública. No 1. Santiago de Chile.

Grindle, M. (1997) “The good government imperative. Human Resources, organizations and institutions", en Merilee S. Grindle (comp) Getting good government. Capacity building in the public sectors of developing countries. Boston, Harvard University Press, 1997: 3-28.

Isuani, F. (2005). "Redes intergubernamentales para la implementación de programas sociales”. X Congreso Internacional del CLAD sobre la reforma del Estado y de la Administración Pública. Santiago de Chile. 18 al 21 de Octubre. 2005.

Jordana, J. (2001), Relaciones Intergubernamentales y descentralización en América Latina: una perspectiva institucional. Documento de Trabajo I-22EU, BID / INDES, Washington DC.

Manor, J. (1999) The Political Economy of Democratic Decentralization. The World Bank, Washington DC.

McIvor, R., McHugh, M. y Caddden, C. (2002): “Internet technologies: supporting transparency in the public sector", The International Journal of Public Sector Management, 15 (3) 3:170-187.

Midaglia, C.; Castillo, M; Freigedo; M. (2009) Relevamiento de iniciativas descentralizadas y desconcentradas de la Administración central del Estado uruguayo. Convenio OPP ("Programa Uruguay Integra") y la Asociación Pro - Fundación para las Ciencias Sociales. Mujica, P. (2005) La participación ciudadana en relación con la gestión pública. Corporación Participativa. Santiago, Chile.

Musgrave, P. (1991) Merits and Demerits of Fiscal Competition. Rémy Prud'homme, ed. Francia.

Oates, Wallace (1977). Federalismo fiscal. Instituto de Estudios de Administración Local, Madrid.

Oszlak, Oscar y Orellana, Edgardo (2001) "El análisis de la capacidad institucional: aplicación de la metodología SADCI”, en: www.oscaroszlak.org.ar/links.php. Fecha de acceso: julio y agosto de 2011.

Ospina, S. (2002) “Construyendo capacidad institucional en América Latina: el papel de la evaluación como herramienta modernizadora”, ponencia presentada en el VII Congreso Internacional del CLAD sobre la Reforma del Estado y de la Administración Pública, Lisboa, Portugal, octubre de 2002.

Ramos, C. (2009) Radiografía del cambio de paradigma en la administración pública. Instituto de Ciencia Política, UDELAR. Uruguay.

Repetto, Fabian (2004) Capacidad estatal: requisito para el mejoramiento de la política social en América Latina. Serie de documentos de trabajo del INDES, Washington, Banco Interamericano de Desarrollo.

Rosas Huerta, Angélica (2008) "Una ruta metodológica para evaluar la capacidad institucional”. Revista Política y Cultura, número 30: 119-134, UAM, Xochimilco. DF, México. 
Royo, S. (2008) El gobierno electrónico en la rendición de cuentas de la Administración Local. Instituto de Estudios Fiscales. Madrid.

Samuelson, P. (1954). "The pure theory of public expenditure". Review of Economics and Statistics, Vol. XXXVI, n.4: 387-389.

Sour-Vargar, L. (2007) "Evaluando al gobierno electrónico: avances en la trasparencia de las finanzas públicas estatales", Economía, sociedad y territorio, VI (23):613-654. El Colegio mexiquense, México.

Vergara, R. (2010) “Introducción”, en:Vergara, R. (comp.) “Organizaciones e Instituciones”, BBA Pdf, Siglo XXI editores, México, 2010: 2-30.

Willis, E., Garman, C. y Haggard, S. (1999): “The politics of decentralization in Latin America", Latin American Research Review. Vol. 34, No 1: 7-56.

Weiss, Linda (1998) The myth of the powerless state. Ithaca, NY: Cornell University Press. Wright, D. (1998). "Para entender las relaciones intergubernamentales", Serie: Nuevas lecturas de política y gobierno, Ed. Fondo de Cultura Económica, México. 